\title{
Multimodality imaging of exuberant mitral annular calcification in a patient presenting with transient ischaemic attack
}

\author{
'S Vijayan, ${ }^{2} \mathrm{P}$ Fielding, ${ }^{3} \mathrm{~B}$ Kahan, ${ }^{4} \mathrm{~A}$ Ionescu \\ ${ }^{1}$ Specialist Registrar in Cardiology; ${ }^{2}$ Consultant Radiologist; ${ }^{3}$ Consultant Physician; ${ }^{4}$ Consultant Cardiologist, Morriston Hospital, Swansea, UK
}

\begin{abstract}
We report an unusual case of calcification of mitral valve annulus imaged with multiple non-invasive modalities in a patient who suffered a transient ischaemic attack, probably from thrombus overlying the mitral annular calcification. Both this mode of presentation and the imaging features of the annular calcification were relatively unusual, and the images obtained are remarkably clear and diagnostic.
\end{abstract}

KEYWORDS Anticoagulation, caseous mitral annular calcification, embolism, multimodality imaging, stroke, transient ischaemic attack
Correspondence to $\boldsymbol{A}$ lonescu, Morriston Cardiac Centre, Morriston Hospital, Swansea SA6 6NL, UK

tel. $+44(0) 1792703195$

e-mail adrian.ionescu@wales.nhs.uk

DECLARATION OF INTERESTS No conflict of interests declared.

\section{CASE REPORT}

An 8I-year-old woman presented to the emergency department with sudden onset weakness of the right side of the body, which had caused her to fall through a glass door and injure her arm. She also had expressive dysphasia. The patient had a previous medical history of hypertension, hypercholesterolaemia and spondylosis. More than a decade ago she had been labelled as suffering from 'angina', based on asymptomatic ST-segment depression during stage 2 of a Bruce protocol. There was no history of rheumatic fever.

Her weakness and expressive dysphasia resolved completely in 90 minutes. On examination, the arterial pulse was regular and normal in character, blood pressure was $138 / 72 \mathrm{mmHg}$ in the right arm and the heart rate was $80 \mathrm{bpm}$. The patient's electrocardiogram showed sinus rhythm with occasional ventricular ectopic beats. The first heart sound was loud and there was a pansystolic murmur grade $3 / 6$ at the mitral area. Neurological examination performed by the admitting physician was normal.

The patient's chest X-ray was suggestive of left atrial enlargement. A computed tomography (CT) scan of the brain did not show any acute infarct or haemorrhage. The initial diagnosis was transient ischaemic attack, of embolic origin.

A carotid duplex scan did not show significant stenoses in the carotid arteries. A transthoracic echocardiogram (TTE) showed a large (approximately $4 \mathrm{~cm}^{2}$ ), round, echogenic, inhomogeneous mass, located below the posterior leaflet of the mitral valve on its ventricular side and casting a broad acoustic shadow (Figure I). The mass was not independently mobile and impinged mildly on the movement of the mitral leaflet; there was mild central mitral regurgitation.

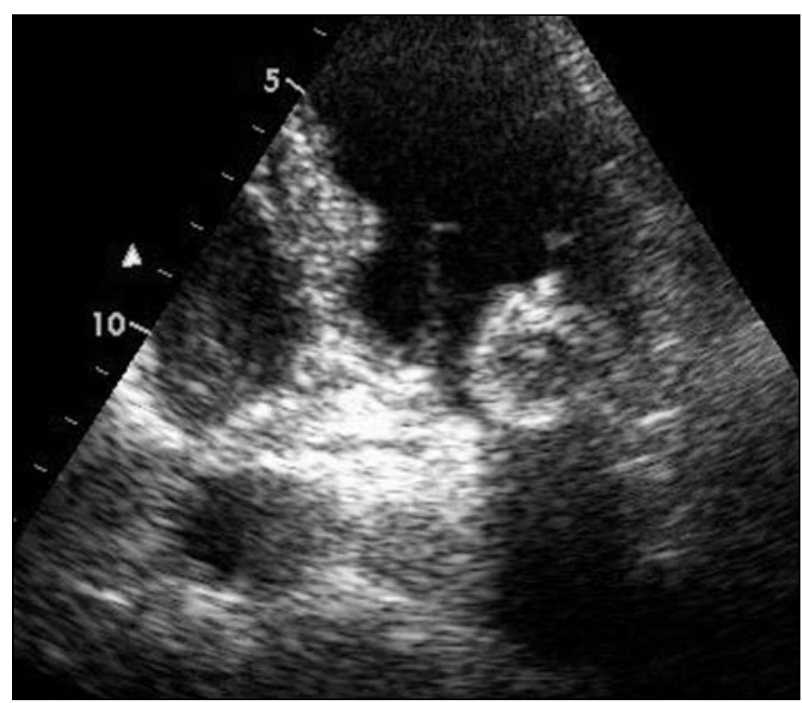

FIGURE I Zoomed, slightly off-axis apical four-chamber view, demonstrating a globular mass with a hypoechoic centre, in the lateral segment of the mitral annulus.

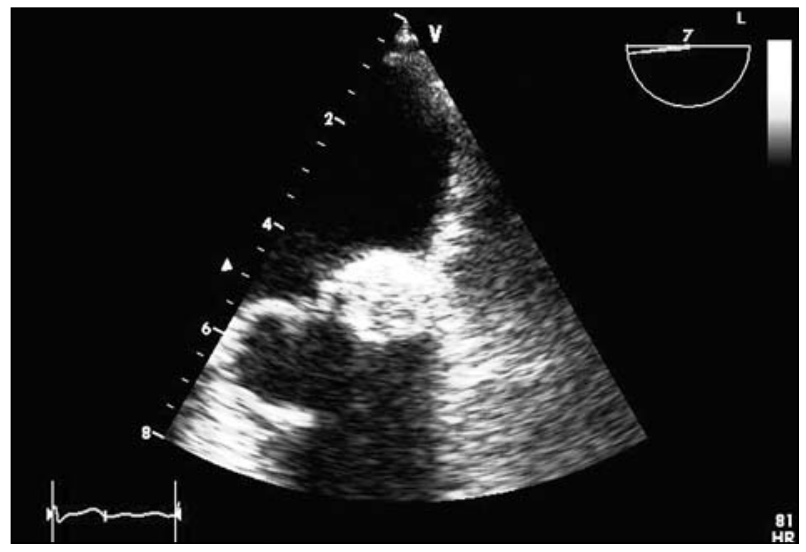

FIGURE 2 Transoesophageal echocardiographic appearances of the mass, in a four-chamber view. The relationship with the mitral valve is better appreciated than on the transthoracic scan. The mass is located on the ventricular aspect of the annulus and distorts the posterior mitral leaflet slightly. 


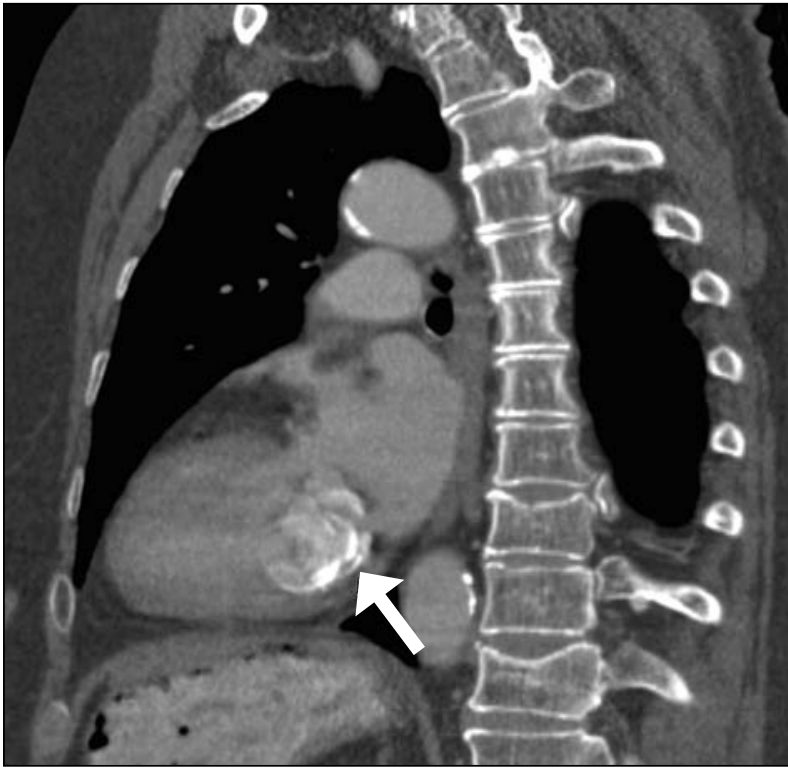

FIGURE 3 Contrast-enhanced computed tomography (CT) scan, where I mm axial images were obtained at $25 \mathrm{~s}$ following injection of iodinated contrast $(300 \mathrm{mg} / \mathrm{ml})$. This oblique sagittal reconstructed view through the mitral valve annulus shows left atrium, left ventricle and densely calcified mitral valve annulus (arrow).

Transoesophageal echocardiography confirmed the findings on TTE (Figure 2). It showed no abnormalities in the left atrial appendage and the inter-atrial septum was intact with no aneurysm or patent foramen ovale.

The heart was imaged further to elucidate the nature of these appearances. On CT there was intense calcification of the mitral annulus and a lobulated $3 \mathrm{~cm}$ mass between the posterior valve leaflet and the posterior wall of the ventricle (Figure 3). On cardiac magnetic resonance imaging (MRI) the mass was intra-ventricular and attached to the posterior mitral valve leaflet and to the posterior wall of the left ventricle (Figure 4). The mass was immobile and hypovascular. The appearances were typical of large calcification of the mitral valve annulus, with tumour or thrombus an unlikely differential.

The patient received anticoagulation with warfarin, with a target international normalised ratio of 2-3. There were no further embolic episodes over three years of followup, and the TTE appearances remained unchanged.

\section{DISCUSSION}

Our patient had an unusual, exuberant type of mitral annular calcification. Caseous calcification of the mitral annulus may mimic the appearance of an intra-cardiac tumour and has been reported before, ${ }^{1-9}$ but while the typical echocardiographic appearance is that of a mass with a central echo-lucent area, ${ }^{8}$ our patient had a more homogenous-appearing mass. Caseous calcification is exceptionally rare, seen in around $0.005 \%$ of TTE studies.

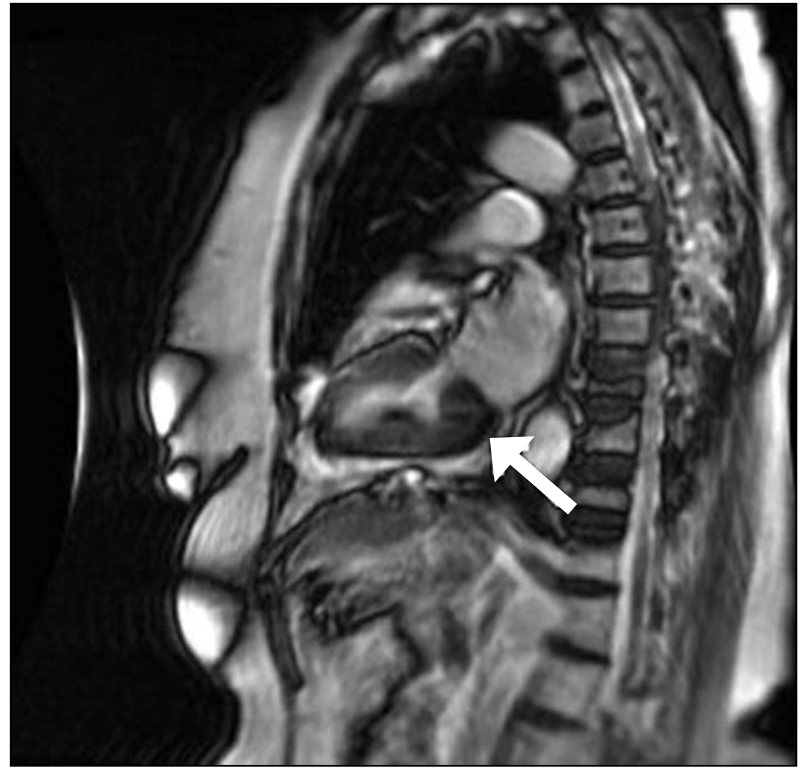

FIGURE 4 Contrast-enhanced magnetic resonance imaging (MRI) scan through the mitral valve annulus. The vertical longaxis image shows left atrium, left ventricle and markedly thickened mitral valve annulus (arrow); the low signal reflects dense calcification here (fast imaging employing steady-state acquisition sequence:TR 2.82 I TEI.2).

Even though caseous calcification is generally benign, there are reports of an increased incidence of stroke and patients have been subjected unnecessarily to surgery when the appearances were interpreted as tumour,' so a correct diagnosis is essential. Several studies have demonstrated an association between stroke and mitral annular calcification (MAC), although a causal link has not been established. ${ }^{10}$ The mechanism of stroke is thought to be embolic, and thrombus overlying MAC has been described, ${ }^{10}$ but calcific embolism has also been suggested.'

We presume thromboembolism was the mechanism of the transient ischaemic attack. The rapid resolution of symptoms and the freedom from further embolism while anticoagulated provide circumstantial support for this hypothesis. It is difficult to image thrombus overlying mitral annular calcification because of acoustic shadowing and because fresh thrombus has a similar echogenicity to blood, so this remains a speculative explanation.

We did not perform a brain MRI scan (which might have identified subtle embolic damage) because we were not convinced it would have changed the treatment. We cannot rule out paroxysmal atrial fibrillation as the substrate for this presentation, but this seems unlikely in the absence of palpitations and with sinus rhythm documented initially and during follow-up. However, we accept that current methods for diagnosing paroxysmal atrial fibrillation are unsatisfactory: one or at most a few 'snapshots' of the heart rhythm are obtained with Holter monitoring. The ongoing Cryptogenic Stroke and underlying Atrial Fibrillation (CRYSTAL) trial will clarify 
whether intensive and invasive monitoring using implantable loop recorders is advisable in patients with cryptogenic stroke. ${ }^{2}$

To the best of our knowledge the use of both CT and MRI to establish this diagnosis has not been reported before. The good outcome with anticoagulation over three years adds to the very limited data about treatment options in this rare setting.

\section{REFERENCES}

I DeVrey EA, Scholte AJ, Krauss XH et al. Intracardiac pseudotumor caused by mitral annular calcification. Eur J Echocardiogr 2006; 7:62-66. http://dx.doi.org/I0.1016/j.euje.2005.02.004

2 Mouton P, Biousse V, Crassard I et al. Ischemic stroke due to calcific emboli from mitral valve annulus calcification. Stroke 1997; 28:2325-26.

3 Fernandes RM, Branco LM, Galrinho A et al. Caseous calcification of the mitral annulus: a review of six cases. Rev Port Cardiol 2007; 26:1059-70.

4 Kato M, Nakatani S, Okazaki $\mathrm{H}$ et al. Unusual appearance of mitral annular calcification mimicking intracardiac tumor prompting early surgery. Cardiology 2006; 106:164-66. http://dx.doi. org/10.1159/000092827

5 De Conti F, Corbetti F, De Conti G et al. Unusual tumor-like calcification of the mitral annulus: diagnosis and tissue characterization by ultrasound, computed tomography and magnetic resonance imaging. Ital Heart J 2005; 6: 160-63.

6 Morgan-Hughes G, Zacharkiw L, Roobottom C et al. Tumor-like calcification of the mitral annulus: diagnosis with multislice computed tomography. Circulation 2003; 107:355-56. http://dx.doi. org/I0.I I6I/0I.CIR.0000047070.50407.D6

\section{CONCLUSION}

Exuberant, tumour-like mitral annular calcification is rare but significant because of the potential for embolic events and because accurate diagnosis avoids inappropriate surgery. Echocardiography is the usual screening test that suggests the diagnosis, but modern multimodality imaging is helpful for confirming it.
7 Harpaz D,Auerbach I,Vered Z et al. Caseous calcification of the mitral annulus: a neglected, unrecognized diagnosis. J Am Soc Echocardiogr 200 I; | 4:825-31. http://dx.doi.org/I0.I067/mje.200I. I I I877

8 Deluca G, Correale M, Leva R et al. The incidence and clinical course of caseous calcification of the mitral annulus: a prospective echocardiographic study. J Am Soc Echocardiogr 2008; 21:828-33. http://dx.doi.org/10.1016/j.echo.2007.12.004

9 Teja K, Gibson RS, Nolan SP. Atrial extension of mitral annular calcification mimicking intracardiac tumour. Clin Cardiology 1987; 10:546-8. http://dx.doi.org//0.1002/clc.49601009/8

10 Stein JH, Soble JS.Thrombus associated with mitral valve calcification: a possible mechanism for embolic stroke. Stroke 1995; 26:1697-9. http://dx.doi.org/I0.I I6I/0I.STR.26.9.1697

I I Benjamin EJ, Plehn JF, D'Agostino RB et al. Mitral annular calcification and the risk of stroke in an elderly cohort. N Engl J Med 1992; 327:374-9. http://dx.doi.org/I0.I056/NEJMI 99208063270602

12 Sinha AM, Diener HC, Morillo CA et al. Cryptogenic Stroke and underlying Atrial Fibrillation (CRYSTAL AF): design and rationale. Am Heart J 2010; I60:36-4I. http://dx.doi.org/I0.10I6/j.ahj.20I0.03.032 